Primeira submissão em 17/06/03 Última submissão em 15/03/04 Aceito para publicação em 01/04/04 Publicado em 20/08/04

\title{
Prevalência de fatores de risco de doença arterial coronária em funcionários de hospital universitário e sua correlação com estresse psicológico
}

\author{
Prevalence of risk factors of coronary artery disease in university hospital \\ employees and the correlation with psychological stress
}

Geny Aparecida Cantos'; Maria de Fátima S. Duarte²; Rosilene Linhares Dutra3 ; Cláudia de Souza Marques da Silva ${ }^{4}$; Carmen Dolores de Araújo Waltrick; ${ }^{4}$ Maria da Graça Balen ${ }^{5}$; Elizabeth Hermes ${ }^{6}$; Aldir Roberto Perozin ${ }^{7}$

\section{unitermos} Doença arterial coronária

Estresse psicológico

Fatores de risco

\section{resumo}

Objetivo: O objetivo do presente estudo foi analisar os diferentes fatores de risco (FR) para doença arterial coronária (DAC) em funcionários voluntários do Hospital Universitário, considerando o efeito do estresse psicológico. Métodos: Participaram deste estudo 52 voluntários (41 mulheres e 11 homens) que foram submetidos a exame antropométrico, aferição da pressão arterial e exame clínico. Foram realizadas também coletas capilares para verificar os níveis de colesterol total (CT), HDL-colesterol (HDL), triglicérides (TG) e LDL-colesterol (LDL), sendo calculados os índices de massa corporais (IMC) e a relação cintura/quadril (RCQ). Foi também aplicado um questionário de estresse. Os diferentes FR para DAC foram quantificados e avaliados. Resultados: Dos indivíduos que participaram desta pesquisa 73,1\% eram estressados, $32,7 \%$ eram hipertensos e $36,5 \%$ apresentaram CT $>200 \mathrm{mg} / \mathrm{dl}$. As mulheres eram mais estressadas que os homens $(p<0,05)$ e os homens apresentavam proporcionalmente menor $\mathrm{HDL}<40 \mathrm{mg} / \mathrm{dl}$ do que as mulheres $(p<0,05)$. Os pacientes estressados apresentaram CT $>200 \mathrm{mg} / \mathrm{dl}(p<0,05)$. O risco coronariano para DAC (RDAC) foi baixo para $53,8 \%$ dos pacientes, médio para $23,1 \%$, moderado para $21,2 \%$ e alto para $2,6 \%$. O risco quadril/cintura (FRCQ) foi alto em $15 \%$ dos indivíduos estressados, em $35,5 \%$ dos hipertensos e em 10,5\% dos com CT > 200mg/dl. O risco massa corporal (FRMC) foi baixo na maioria dos pacientes estressados, hipertensos e com CT > 200mg/dl. Conclusões: Este estudo mostra a importância do estresse psicológico na avaliação dos diferentes fatores de risco para DAC, sendo que esse conhecimento é de grande relevância na intervenção preventiva para essa doença.

\section{abstract}

Objective: The present work aimed to evaluate the different risk factors (RF) of coronary artery disease (CAD) in a university hospital volunteer employees, regarding the correlation with psychological stress. Methods: Fifty-two volunteers participated in the study (41 females and 11 males), which included anthropometric measurement, clinical examination, and measurement of the arterial blood pressure. Arterial blood was collected from them to quantify the levels of total cholesterol, $H D L$-cholesterol (HDL), triglycerides (TG), and LDL-cholesterol (LDL). The body mass index (BMI), and waist-to-hip ratio (WHR) were determined. The participants have also answered a questionnaire regarding stress. The different $R F$ were quantified and evaluated. Results: From the participants, 73.1\% were considered stressed, $32.7 \%$ were hypertenses, and $36.5 \%$ had TC $>200 \mathrm{mg} \mathrm{dl}^{-1}$. Females were significantly more stressed than man $(p<.05)$, and proportionally males had more $H D L<40 \mathrm{mg} \mathrm{dl}^{-1}$ than females $(p<.05)$. The stressed people also presented TC above $200 \mathrm{mg} \mathrm{d}^{-1}(p<.05)$. The coronary artery disease risk was low for $53.8 \%$ of the people, medium for $23.1 \%$, moderate for $21.2 \%$, and high for $2.6 \%$. The waist-to-hip risk was high in $15 \%$ of the stressed patients, in 35,5\% of hypertenses, and in $10.5 \%$ in the ones with TC > $200 \mathrm{mg} / \mathrm{dl}$. The body mass risk was considered low for most of the stressed, hypertenses, and the ones with TC > $200 \mathrm{mg} \mathrm{dl}^{-1}$. Conclusion: This study shows the importance of the psychological stress in the evaluation of different RF for CAD and this knowledge is very important in the preventive intervention for this disease. key words Coronary heart disease Psychological stress Coronary heart risk factors

1. Professora-doutora do Centro de Ciências da Saúde da Universidade Federal de Santa Catarina (UFSC).

2. Professora-doutora do Centro de Desportes da UFSC.

3. Aluna do Curso de Doutorado em Química da UFSC.

4. Cardiologista do Hospital Universitário (HU) da UFSC.

5. Nutricionista do HU/UFSC.

6. Bioquímica do HU/UFSC.

7. Mestre em Informática e profissional voluntário. 


\section{Introdução}

A doença arterial coronariana (DAC) é um problema de crescente prevalência, principalmente nos grandes centros e nas populações de faixa etária mais elevada, ocorrendo um aumento de mortalidade em função da mesma ${ }^{(11,33,37,39)}$.

A doença aterosclerótica é considerada multifatorial, e a sua prevenção passa pela identificação do conjunto dos fatores de risco (FR). Estes se dividem em modificáveis e não-modificáveis. Os não-modificáveis incluem a idade, o sexo e a história familiar positiva para DAC precoce. Entre os modificáveis estão a dislipidemia, o diabetes, o tabagismo, o sedentarismo, a hipertensão arterial e a obesidade. A prevenção tem sido baseada no conhecimento dos fatores de risco modificáveis $(6,30,45,46)$.

A percepção entre relação de riscos à saúde e estresse também tem sido relatada. Trabalhos sugerem que o estresse psicológico esteja relacionado ao desenvolvimento de doenças cardiovasculares. Heslop et al.(14), em 2002, exploraram a associação entre satisfação no trabalho e doenças cardiovasculares. Esses mesmos autores, em uma outra publicação, sugerem que insuficiência de sono esteja associada a um aumento de risco de mortalidade ${ }^{(15)}$. Por outro lado Vögele ${ }^{(44)}$, em 2002, concluiu que a combinação de fatores de risco, incluindo os psicológicos, contribuem para o melhor entendimento da morbidade e mortalidade cardiovascular.

A elevação da pressão arterial é um FR importante para DAC, pois danifica o revestimento das artérias e acelera o desenvolvimento de ateroma ${ }^{(32)}$. $O$ risco de hipertensão tem sido associado ao estresse mental ${ }^{(1)}$. Por outro lado, pesquisas mostram que cerca de $50 \%$ dos idosos acima de 65 anos apresentam hipertensão sistólica isolada, com aumento de eventos vasculares encefálicos e cardíacos ${ }^{(19)}$. As mulheres são relativamente protegidas de doenças cardiovasculares, em geral, e de hipertensão arterial, em particular, durante a fase reprodutiva. Porém após a menopausa, a incidência de infarto agudo do miocárdio aumenta dramaticamente, e hipertensão arterial torna-se progressivamente mais freqüente e mais intensa em mulheres que em homens ${ }^{(29)}$.

A hipertensão pode também estar associada a dislipidemia, a intolerância à glicose, a obesidade, entre outros ${ }^{(5,}$ ${ }^{40)}$. A associação entre obesidade e hipertensão está bem estabelecida, sendo que nas estatísticas americanas a prevalência de hipertensão nos obesos é de $35,5 \%{ }^{(13)}$. As anormalidades sugeridas para explicar a alta suscetibilidade dos obesos em desenvolver hipertensão compreendem a retenção de sódio, a desregulação nos hormônios sais mo- duladores, o aumento do volume plasmático e do débito cardíaco, a hiperinsulinemia e a resistência insulínica, além de aumento da atividade simpática ${ }^{(33)}$.

A obesidade, avaliada pelo índice de massa corporal (IMC), está freqüentemente associada a outros FR para saúde, e mais especificamente para doenças cardiovasculares; uma forma prática e eficiente de se avaliar a distribuição de gordura na região abdominal (gordura visceral) é também a relação cintura/quadril, cujos valores desejáveis são de < 0,9 para homens e $<0,8$ para mulheres ${ }^{(13)}$.

A glicemia é hoje considerada um FR independente, elevando a DAC para alto risco tanto nos pacientes diabéticos dependentes de insulina quanto nos não-dependentes de insulina. Recomenda-se que todos os indivíduos acima de 45 anos façam um rastreamento para diabetes ${ }^{(9,24)}$. $\mathrm{O}$ diabetes e as dislipidemias, quando coexistentes, aceleram as alterações vasculares e o processo de envelhecimento dos vasos $^{(33)}$, levando a alterações significativas nos níveis séricos das frações lipídicas, do colesterol e dos triglicérides ${ }^{(12)}$.

$\mathrm{O}$ estresse também provoca o aumento de LDL-colesterol (LDL) e a diminuição da fração HDL-colesterol (HDL), sugerindo um aumento de doenças cardiovasculares ${ }^{(4,38)}$. O estresse também afeta a pressão arterial com a estimulação do sistema nervoso simpático, produzindo aumento da freqüência cardíaca e da força contrátil dos batimentos cardíacos, bem como da resistência periférica, aumentando assim o risco de $\operatorname{DAC}^{(4,20,35)}$. No aparelho circulatório, o estresse e o frio são fatores que aumentam a atividade simpática e levam à liberação de adrenalina, promovendo taquicardia e uma vasoconstrição( ${ }^{(20)}$.

O consumo de cigarros constitui um fator de risco independente bem estabelecido, tanto em homens quanto em mulheres, no que se refere à DAC. O tabagismo na formação e na evolução da placa aterosclerótica é capaz de produzir lesões endoteliais de forma direta, levando a uma maior oxidação da LDL, reduzindo a produção de HDL. A diminuição da resposta vasodilatadora dependente do endotélio durante o esforço pode, inclusive, induzir vasoconstrição em repouso. Esse efeito é observado tanto nos fumantes ativos quanto nos passivos ${ }^{(33,42)}$. Contudo, os tabagistas apresentam, de forma geral, aproximadamente o dobro da taxa geral de mortalidade e quase o dobro de mortalidade por causas coronarianas, quando comparados a não-fumantes ${ }^{(8,42)}$.

Como a DAC é multifatorial, é muito importante estimar o risco dessa doença para um determinado indivíduo, considerando todos os FR presentes simultaneamente. Os índices de risco são os elementos analíticos principais, que, 
além de indicar a importância dos FR na enfermidade, constituem também um instrumento prático de prevenção, permitindo ao médico calcular o risco de um paciente em relação ao que seria considerado ideal para aquele indivíduo em questão (mesmo sexo e idade) ${ }^{(2,41)}$.

Dentro desse contexto, este trabalho teve por objetivo avaliar os diferentes FR e a probabilidade futura para DAC em funcionários voluntários do Hospital Universitário (HU) da Universidade Federal de Santa Catarina (UFSC), em Florianópolis.

\section{Material e método}

Participou deste estudo uma amostra de 52 funcionários do Hospital Universitário (HU) da Universidade Federal de Santa Catarina, sendo que os mesmos foram voluntários que transitavam em jejum pelo hall (HU), entre os dias 8 e 11 de maio de 2001. A faixa etária dessa população variou entre 18 e 60 anos, sendo constituída de $78,85 \%$ de mulheres e $21,15 \%$ de homens. Todos os participantes tiveram o devido esclarecimento sobre a presente pesquisa e assinaram o termo de consentimento informado, para que os dados pudessem ser publicados.

Esses funcionários foram submetidos a exame antropométrico (massa corporal - kg, estatura - $\mathrm{m}$, perímetro da cintura e quadril $-\mathrm{cm}$ ), aferição da pressão arterial e exame clínico. O IMC foi obtido pela divisão da massa corporal, em quilogramas, pelo quadrado da estatura, em metros $\left(\mathrm{IMC}=\right.$ massa $(\mathrm{kg}) /$ estatura $\left.^{2}\right)$. Foram considerados com sobrepeso tanto homens quanto mulheres com IMC > $25 \mathrm{~kg} / \mathrm{m}^{2} \mathrm{e}<30 \mathrm{~kg} / \mathrm{m}^{2}$, e obesos aqueles com o IMC superior a $30 \mathrm{~kg} / \mathrm{m}^{2}$. O risco massa corporal (RFMC) foi considerado normal (classe 0) quando o IMC ficou entre 20 e 24; baixo (classe 1), entre 25 e 29; moderado (classe 2), entre 30 e 34; muito alto (classe 3), entre 35 e 39 e > ou igual a 40, muito alto (classe 4).

O diagnóstico da topografia da circunferência abdominal foi feito pela medida do perímetro da cintura e do quadril e conseqüentemente da divisão de um pelo outro (razão cintura/quadril). A medição da cintura foi feita na região mais estreita do abdômen e a medição do quadril foi feita na região trocantérica. Os pontos de corte de normalidade considerados foram $80 \mathrm{~cm}$ e $94 \mathrm{~cm}$, respectivamente para mulheres e homens. O ICQ foi considerado baixo, masculino $<0,9$ e feminino $<0,8$; moderado, masculino entre 0,9 e 0,95; alto, masculino $>0,95$ e feminino $>0,85$. O risco quadril/cintura (FRCQ) foi considerado: 0 , normal; 1, baixo; 2, moderado; e 3, alto.
Os valores de referência utilizados para definição de hipertensão arterial (HA) foram os mesmo utilizados pela American College of Sports Medicine ${ }^{(2)}$. As medidas foram realizadas no braço direito, estando o indivíduo sentado. A pressão arterial foi medida por meio de um esfigmomanômetro de coluna de mercúrio, considerando a primeira buIha de Korotkow para sistólica e a última, para diastólica.

Considerou-se também a história do uso do tabaco, sendo fumantes aqueles trabalhadores que, na ocasião, fumavam qualquer quantidade de tabaco todos os dias. Para o rastreamento de diabetes mellitus utilizou-se apenas o inquérito clínico, ou seja: diabético ou não-diabético.

Na mesma ocasião foi realizada uma coleta capilar para verificação dos níveis de lípides, analisada pelo aparelho Cholestech L.D.X Analyser, que combina metodologia enzimática e tecnologia de fase sólida para separar colesterol total (CT), HDL e triglicérides (TG). Os valores de LDL foram calculados utilizando a fórmula de Friedwald.

Os indivíduos também responderam a um questionário sobre estado de estresse proposto por Lipp et al.(21), em 1986. Por esse questionário, classifica-se o indivíduo em sete estados de risco de estresse: 1) bem-estar positivo; 2) baixa positividade; 3 ) marginal; 4) problema de estresse; 5) sofrimento; 6) sério sofrimento; 7) severo sofrimento.

Para o risco coronariano para doença arterial coronariana (RDAC) os escores de risco de Framingham foram utilizados como ferramenta de auxílio de estratificação do risco de eventos coronarianos, onde foram calculados o risco absoluto de eventos em dez anos (3, alto risco com $\geq 20 \%$; 2, médio risco de evento $>10 \%$, porém menor do que $20 \%$ e 1, baixo risco < que 10\%) segundo resumo das III Diretrizes Brasileiras sobre Dislipidemia e Diretriz de Prevenção da Aterosclerose da Sociedade Brasileira de Cardiologia(41).

Para análise dos dados foi calculada a estatística básica (média, desvio padrão, percentual), teste $t$ de Student e qui-quadrado com nível de significância de $5 \%$.

\section{Resultados}

Considerando as informações referentes ao grupo estudado, pode-se observar que a população em estudo obteve resultado satisfatório no que diz respeito às análises sangüíneas, mas não em relação ao IMC, onde todo o grupo se apresentou com sobrepeso. Podem-se notar algumas diferenças significativas em relação ao sexo, no que diz respeito à fração $\mathrm{HDL}$ e ao $\mathrm{ICQ}$, sendo que as mulheres apresentaram valores maiores de HDL e valores menores de ICQ comparados aos homens (Tabela 1). Em relação ao 
TG somente três mulheres apresentaram os valores acima de $200 \mathrm{mg} / \mathrm{dl}$ (Tabela 2).

Outros FR analisados foram a hipertensão arterial, o estresse, o DM e o tabagismo. Pode-se notar que a maioria da população $(73,1 \%)$ em estudo apresentou problemas ou sofrimento quanto ao estresse, sendo que proporcionalmente as mulheres mostraram-se significativamente mais estressadas que os homens $(80,5 \%$ e $45,5 \%$, respectivamente). Por outro lado, uma boa parcela do grupo estudado apresentou hipertensão, sendo que as diferenças observadas entre os sexos não foram significativas. Pode-se notar que $9,6 \%$ dos funcionários que participaram dessa pesquisa eram tabagistas e 5,8\% eram diabéticos (Tabela 2).

A Tabela 3 mostra a relação de funcionários que eram estressados e que também eram hipertensos e dislipidêmicos. Pode-se notar que $82,4 \%$ dos indivíduos estressados também eram hipertensos e que $89,5 \%$ dos estressados tinham CT > 200mg/dl (associação significativa estatisti-

\section{Resultados das análises sangüineas e das medidas antropométricas em funcionários}

Tabela 1 do HU/UFSC

\begin{tabular}{cccc}
\hline Variáveis & Sexo feminino & Sexo masculino & Total \\
& $(n=41)$ & $(n=11)$ & $(n=52)$ \\
CT mg/dl & $193,27^{\mathrm{a}} \pm 38,92^{\mathrm{b}}$ & $186,36^{\mathrm{a}} \pm 40,43^{\mathrm{b}}$ & $191,81^{\mathrm{a}} \pm 38,94^{\mathrm{b}}$ \\
& $100-306^{\mathrm{c}}$ & $132-256^{\mathrm{c}}$ & $100-306^{\mathrm{c}}$ \\
HDL mg/dl & $56,02^{\mathrm{a}} \pm 13,45^{\mathrm{b}}$ & $41,64^{\mathrm{a}} \pm 6,56^{\mathrm{b}}$ & $52,98^{\mathrm{a}} \pm 13,62^{\mathrm{b}}$ \\
& $34-100^{\mathrm{c}}$ & $33-53^{\mathrm{c}}$ & $33-100^{\mathrm{c}}$ \\
LDL mg/dl & $114,29^{\mathrm{a}} \pm 33,23^{\mathrm{b}}$ & $125,46^{\mathrm{a}} \pm 32,93^{\mathrm{b}}$ & $116,65^{\mathrm{a}} \pm 33,16^{\mathrm{b}}$ \\
& $19-181^{\mathrm{c}}$ & $82-184^{\mathrm{c}}$ & $19-184^{\mathrm{c}}$ \\
TG mg/dl & $94,76^{\mathrm{a}} \pm 62,55^{\mathrm{b}}$ & $97,09^{\mathrm{a}} \pm 39,36^{\mathrm{b}}$ & $95,25^{\mathrm{a}} \pm 58,08^{\mathrm{b}}$ \\
& $44-324^{\mathrm{c}}$ & $45-164^{\mathrm{c}}$ & $44-324^{\mathrm{c}}$ \\
CT/HDL & $3,68^{\mathrm{a}} \pm 7,37^{\mathrm{b}}$ & $4,54^{\mathrm{a}} \pm 1,16^{\mathrm{b}}$ & $3,86^{\mathrm{a}} \pm 1,36^{\mathrm{b}}$ \\
& $1,39-7,85^{\mathrm{c}}$ & $3,1-6,03^{\mathrm{c}}$ & $1,39-7,85^{\mathrm{c}}$ \\
IMC $\mathrm{kg} / \mathrm{m}^{2}$ & $27,32^{\mathrm{a}} \pm 5,59^{\mathrm{b}}$ & $26,94^{\mathrm{a}} \pm 4,4^{\mathrm{b}}$ & $27,24^{\mathrm{a}} \pm 5,32^{\mathrm{b}}$ \\
& $16,36-38,95^{\mathrm{c}}$ & $20,45-34,17^{\mathrm{c}}$ & $16,36-38,95^{\mathrm{c}}$ \\
ICO & $0,79^{\mathrm{a}} \pm 0,06^{\mathrm{b}}$ & $0,88^{\mathrm{a}} \pm 0,06^{\mathrm{b}}$ & $0,81^{\mathrm{a}} \pm 0,07^{\mathrm{b}}$ \\
& $0,69-0,92^{\mathrm{c}}$ & $0,75-0,98^{\mathrm{c}}$ & $0,69-0,98^{\mathrm{c}}$ \\
\hline
\end{tabular}

${ }^{a}$ Média; $b$ desvio-padrão; 'amplitude.

\section{Tabela 2 Distribuição de diferentes FR para DAC em funcionários do HU/UFSC}

\begin{tabular}{lccccc}
\hline Parâmetros & \multicolumn{2}{c}{ Sexo feminino } & \multicolumn{2}{c}{ Sexo masculino } & Total \\
considerados & $(n=41)$ & $\%$ & $(n=11)$ & $\%$ & $(n=52)$ \\
\hline Hipertensão & 13 & 31,7 & 4 & 36,4 & 32,7 \\
Estresse & 33 & 80,5 & 5 & 45,5 & $73,1^{*}$ \\
Diabetes mellitus & 3 & 7,3 & - & - & 5,8 \\
Tabagismo & 3 & 7,3 & 2 & 18,2 & 9,6 \\
CT $>200 \mathrm{mg} / \mathrm{dl}$ & 15 & 36,6 & 4 & 36,4 & 36,5 \\
HDL $<40 \mathrm{mg} / \mathrm{dl}$ & 3 & 7,3 & 5 & 45,5 & $15,4^{*}$ \\
$\mathrm{LDL}>130 \mathrm{mg} / \mathrm{dl}$ & 11 & 26,8 & 5 & 45,5 & 30,8 \\
$\mathrm{TG}>200 \mathrm{mg} / \mathrm{dl}$ & 3 & 7,3 & - & - & 5,8 \\
\hline${ }^{*} p<0,05\left(\chi^{2}=5,51 ; \chi^{2}=9,69\right)$. & & & & &
\end{tabular}




\begin{tabular}{ccc} 
& \multicolumn{2}{c}{$\begin{array}{l}\text { Associação entre hipertensão, } \\
\text { dislipidemia e estresse em } \\
\text { funcionários do HU/UFSC }\end{array}$} \\
\hline Tabela 3 & Funcionários estressados \\
\hline Parâmetros & $n$ & $\%$ \\
considerados & 14 & 82,4 \\
Hipertensão & 17 & $89,5^{*}$ \\
CT $>200 \mathrm{mg} / \mathrm{dl}$ & 6 & 75 \\
$\mathrm{HDL}<40 \mathrm{mg} / \mathrm{dl}$ & 14 & 77,8 \\
$\mathrm{LDL}>130 \mathrm{mg} / \mathrm{dl}$ & &
\end{tabular}

${ }^{*} p<0,05\left(\chi^{2}=4,091\right)$.

camente); $75 \%$ tinham $\mathrm{HDL}<40 \mathrm{mg} / \mathrm{dl} \mathrm{e} 77,8 \%$ apresentaram LDL $>130 \mathrm{mg} / \mathrm{dl}$.

A Tabela 4 mostra a associação dos FR (estresse, HA e CT) em relação à probabilidade futura de doença coronária (RDAC). Nota-se que $58,9 \%$ dos funcionários que apresentaram HA e $36,9 \%$ dos que tinham CT > 200mg/dl tiveram FRDC moderado e alto, respectivamente. Pode-se observar ainda que $55 \%$ dos funcionários estressados tinham RDAC baixo e que apenas $2,6 \%$ tinham RDAC alto, mas nenhuma das proporções foi estatisticamente significante.

A Tabela 5 mostra que da amostra total, $59,6 \%$ dos funcionários estressados, hipertensos e com CT > 200mg/dl apresentaram baixo FRCQ; $28,8 \%$ tinham risco moderado e $11,5 \%$, risco alto. O FRCQ foi baixo para $55 \%$ dos estressados, moderado para $30 \%$ deles e alto para $15 \%$. Por outro lado, em indivíduos hipertensos, pode-se notar que o FRCQ foi de $35,3 \%$ (baixo); $29,4 \%$ (moderado) e $35,5 \%$ (alto). Em relação ao CT esse risco mostrou-se baixo para $52,6 \%$ dos indivíduos que tinham CT $>200 \mathrm{mg} / \mathrm{dl}$, moderado para $36,8 \%$ e alto para $10,5 \%$.

Na Tabela 6, nota-se que o FMC foi baixo na maioria dos pacientes estressados (com problemas, sofrimento ou sério sofrimento ao estresse), hipertensos e com CT > 200 mg/dl. Por outro lado, observa-se que $35,3 \%$ dos funcionários hipertensos apresentaram FRMC alto.

\section{Discussão}

No Brasil as doenças cardiovasculares são as principais causas de mortalidade, vitimando aproximadamente 300 mil brasileiros ao ano, provocando grandes gastos com assistência médica, sendo prevalentes principalmente nos grandes centros e nas populações de faixa etária mais alta ${ }^{(33,}$ 39). Apesar da enorme evolução que se teve na cardiologia nas últimas décadas, como por exemplo, o crescente conhecimento da fisiologia da aterosclerose com ênfase na placa do ateroma e suas complicações ${ }^{(3,17,22,28)}$, o lan-

\section{Tabela 4 e com CT $>200 \mathrm{mg} / \mathrm{dl}$}

\begin{tabular}{lcccccc} 
RDC & \multicolumn{2}{c}{ Estressados } & \multicolumn{2}{c}{ Hipertensos } & \multicolumn{2}{c}{ CT $>200 \mathrm{mg} / \mathrm{dl}$} \\
& $n$ & $\%$ & $n$ & $\%$ & $n$ & $\%$ \\
1. Baixo & 22 & 55 & 3 & 17,65 & 6 & 31,57 \\
2. Moderado & 9 & 22,5 & 3 & 17,65 & 7 & 36,85 \\
3. Alto & 8 & 20 & 10 & 58,82 & 5 & 26,32 \\
4. Muito alto & 1 & 2,5 & 1 & 5,88 & 1 & 5,26 \\
\hline
\end{tabular}

Associação entre FRCQ, estresse, hipertensão e CT em funcionários do Hospital Universitário, Tabela 5 Florianópolis, 2001

\begin{tabular}{lccccccc}
\hline FRCQ & \multicolumn{2}{c}{ Estressados } & \multicolumn{2}{c}{ Hipertensos } & \multicolumn{2}{c}{ CT $>200 \mathrm{mg} / \mathrm{dl}$} & \multicolumn{2}{c}{ Total } \\
& $\mathrm{n}$ & $\%$ & $\mathrm{n}$ & $\%$ & $\mathrm{n}$ & $\%$ & $\%$ \\
1. Baixo & 22 & 55 & 6 & 35,3 & 10 & 52,6 & 59,6 \\
2. Moderado & 12 & 30 & 5 & 29,4 & 7 & 36,8 & 28,8 \\
3. Alto & 6 & 15 & 6 & 35,5 & 2 & 10,5 & 11,5 \\
\hline
\end{tabular}


Associação entre FRMC, estresse, hipertensão e CT em funcionários do Hospital Universitário,

Tabela 6 Florianópolis, 2001

\begin{tabular}{lcccccc} 
FRMC & \multicolumn{2}{c}{ Estressados } & \multicolumn{2}{c}{ Hipertensos } & \multicolumn{2}{c}{ CT $>200 \mathrm{mg} / \mathrm{dl}$} \\
& $n$ & $\%$ & $n$ & $\%$ & $n$ & $\%$ \\
0. Normal & 2 & 5 & 3 & 17,6 & 4 & 21,1 \\
1. Baixo & 22 & 55 & 6 & 35,3 & 9 & 47,4 \\
2. Moderado & 10 & 25 & 2 & 11,8 & 3 & 15,8 \\
3. Alto & 6 & 15 & 6 & 35,3 & 3 & 15,8
\end{tabular}

çamento de novas drogas no arsenal terapêutico $(26,39,46), 0$ advento da biologia molecular, a engenharia genética ${ }^{(23)}$, entre outros, até o presente não se conseguiu minimizar de forma satisfatória as conseqüências da doença arterial coronariana. Porém, para se obter um resultado satisfatório real devem-se considerar aqueles atributos que estatisticamente contribuem para o desenvolvimento da DAC e deve-se intervir de maneira concreta nos chamados fatores de risco (FR) para $\mathrm{DAC}^{(23,34)}$.

Alguns FR, como sexo e idade, são fatores não-modificáveis, que não permitem qualquer tipo de ação preventiva. De maneira geral, algumas lesões ateromatosas vão se desenvolvendo com o passar do tempo e os homens são mais afetados do que as mulheres, sobretudo quando são mais jovens ${ }^{(39)}$. A Tabela 1 mostra o perfil das análises sangüíneas e das medidas antropométricas dos funcionários do Hospital Universitário, considerando as diferenças ocorridas entre os sexos. Nota-se que a fração $\mathrm{HDL}$ e a relação $\mathrm{CT} / \mathrm{HDL}$ foi maior nos homens. O IMC mostrou que os funcionários de ambos os sexos estavam com sobrepeso.

Neste trabalho, pode-se notar que, de maneira geral, as mulheres apresentaram-se significativamente mais estressadas que os homens (Tabela 2). De fato, pesquisas mostram que as mulheres estão cada vez mais expostas ao estresse $\mathrm{e}$ que há um significante aumento do uso de tabaco por parte das mesmas, com aumento da pressão sistólica e níveis de colesterol $^{(10,36)}$. Assim, o interesse dos cardiologistas pelo estresse é justificável, pois o mesmo pode causar aumento dos níveis de $\mathrm{CT}^{(40,43)}$, de glicose, da obesidade e da hipertensão arterial, o que poderia levar a isquemia miocárdica, arritmias cardíacas e morte súbita ${ }^{(27,33)}$.

É lógico que o estresse emocional não é sozinho o fator causal da hipertensão arterial. Há muitas pessoas que não têm estresse e que sofrem de hipertensão, como também há outras que têm estresse e não são hipertensas. Não há dúvida, porém, de que existe uma correlação entre estresse e hipertensão(20,35). Antes dos 40 anos os homens também têm maior tendência à hipertensão, porém nos idosos a prevalência é maior nas mulheres ${ }^{(5)}$. Portanto, a idade é um fator a ser considerado no controle da pressão sangüínea, e medidas como o tratamento anti-hipertensivo e/ou exercício físico e dietoterapia parecem ter grande importância na prevenção de eventos cardiovasculares $(6,17,18,25,30)$. Outros FR, particularmente a hipercolesterolemia, estão freqüentemente presentes em pacientes hipertensos ${ }^{(32,46)}$.

Sabe-se que, em indivíduos com idade abaixo de 25 anos, um valor de CT superior a $200 \mathrm{mg} / \mathrm{dl}$ ou de TC acima de $150 \mathrm{mg} / \mathrm{dl}$ indica hiperlipidemia. Porém a hiperlipidemia representa um risco mais significativo para DAC em indivíduos com idade inferior a 50 anos $^{(36)}$, sendo cerca de três a quatro vezes mais freqüente nos homens do que nas mulheres até os 55 anos. Após os 75 anos de idade, as incidências se igualam em ambos os sexos ${ }^{(10,39)}$. Por outro lado, outras pesquisas assinalam a existência de correlações entre as frações HDL e LDL e as placas ateroscleróticas ${ }^{(44)}$. Nesta pesquisa pode-se notar que as mulheres também tiveram o CT e a fração LDL mais elevados que os homens, mas essas diferenças não foram significativas. Contudo, a fração HDL foi mais elevada nas mulheres, o que leva a considerar que talvez estas sejam menos sedentárias que os homens. Em relação à idade, observou-se que dois funcionários (um homem e uma mulher) não apresentavam problemas de estresse, possuíam TG, CT e frações dentro dos valores de referência. No entanto, um deles, possivelmente por apresentar a idade menor (18 anos) que o outro paciente, teve um RDAC menor, indicando com isso o aumento de RDAC com o passar dos anos.

Na Tabela 3 pode ser constatado que dos 17 funcionários hipertensos, $82,4 \%$ apresentaram estados de estresse $\geq$ 4, assim como nos 17 funcionários com CT > 200, 89,47\% obtiveram estados de estresse $\geq 4$. Esses dados sugerem que o estresse pode estar correlacionado com a condição de 
hipertensão arterial e também com o aumento dos níveis de $\mathrm{CT}$, com conseqüente risco de esses funcionários apresentarem DAC, conforme mostra a literatura ${ }^{(4,20)}$. Ainda na Tabela 3 , pode-se notar que $75 \%$ dos indivíduos que apresentavam problemas ou sofrimento devido ao estresse tinham $\mathrm{HDL}<$ 40. Na amostra, duas funcionárias apresentaram sofrimento severo devido ao estresse (estado de estresse $=7$ ), sendo que estas foram também as que apresentaram CT elevados (283 e 306mg/dl), elevada relação CT/HDL $(5,05$ e 7,85) e moderado risco coronariano.

O risco para DAC está aumentado em cerca de duas a quatro vezes em pessoas com diabetes mellitus ${ }^{(9,40)}$. Apesar de o número de funcionários ser pequeno nesta pesquisa, observou-se que dois diabéticos tiveram estimado o RDAC $\geq 3$, e os FRCQ e FRMC moderados e altos, respectivamente.

O tabagismo atua na formação e na evolução da placa aterosclerótica e é capaz de produzir lesões endoteliais de forma direta. É dose-dependente, por isso os tabagistas apresentam aproximadamente o dobro da taxa geral de mortalidade e quase o dobro de mortalidade por causas coronarianas quando comparados a não-fumantes ${ }^{(8,42)}$. Neste estudo, como poucos funcionários eram tabagistas, esse parâmetro não ficou em evidência. Apesar disso, pode-se notar que uma funcionária fumante apresentou a seguinte condição: $\mathrm{CT}=223 \mathrm{mg} / \mathrm{dl} ; \mathrm{HDL}=34 \mathrm{mg} / \mathrm{dl} ; \mathrm{LDL}=$ $154 \mathrm{mg} / \mathrm{dl}$; a relação CT/HDL foi de 6,56; o RDAC e o FRCQ foram moderados; o FRMC foi alto. Esses dados sugerem que o cigarro pode contribuir para eventos coronários tal como mostra a literatura ${ }^{(16,31)}$.

$\mathrm{O}$ risco de aterosclerose e suas seqüelas aumentam progressivamente com o aumento da pressão arteria|(5). A pressão alta danifica o revestimento das artérias e acelera 0 desenvolvimento de ateroma. A hipertensão na maioria das vezes vem acompanhada de outros FR (diabetes, tabagismo, obesidade). Neste estudo pode-se comprovar que $64,9 \%$ dos hipertensos apresentavam RDAC $\geq 3$ e FRMC e FRQC entre moderados e altos.

A obesidade é também considerada um outro grande FR para DAC. Três funcionários apresentaram seus parâmetros aumentados: IMCs de 35,4, 35,7 e 34,2kg/m²; ICQ de 0,84, 0,91 e 0,86 e tiveram com isso um RDAC moderado e FRCQ entre moderado e alto.

Assim, por ser um estudo entre funcionários voluntários, a amostra feminina foi mais expressiva que a masculina, o que não reflete o perfil dos funcionários do $\mathrm{HU}$, mas já demonstra a necessidade de valorização para DAC em ambos os sexos. O uso de tabelas como ferramenta de auxílio na estratificação do risco de eventos clínicos coronarianos orienta a estimativa do nível de risco de eventos coronarianos em dez anos, estabelecendo que os indivíduos com risco abaixo de $20 \%$ devem ser orientados a corrigir seus FR e manter seu risco baixo, enquanto indivíduos com risco superior a $20 \%$ devem modificar os FR de forma intensiva ${ }^{(41)}$.

\section{Conclusões}

Mesmo considerando as limitações inerentes a este estudo, esses resultados somam-se a outros trabalhos e assinalam que o estresse psicológico pode ser um fator adicional a ser considerado na avaliação dos diferentes FR para DAC. Dentro desse contexto, nota-se a necessidade de se traçarem estratégias preventivas eficientes por meio da iniciativa colaborativa envolvendo sociedade, instituições e governos, exigindo não só o conhecimento dos FR convencionais e emergentes, mas também seu risco associado e impacto clínico sobre diferentes populações.

\section{Referências}

I. AL'ABSI, M.; EVERSON, S.A.; LOVALLO,W.R. Hypertension risk factors and cardiovascular reactivity to mental stress in young men. International Journal of Psychophysiology, v. 20: p. $155-60,1995$

2. AMERICAN COLLEGE OF SPORTS MEDICINE. ACSM'S Guidelines for Exercise Testing and Prescription. $6^{\text {th }}$ ed. Baltimore: Lippincott Williams \& Wilkins, 2000. 368 p.

3. BORENSTEIN, M.S. (org.) Manual de Hipertensão. Porto alegre: Editora Sagra, 1999.92 p.

4. CALDERON, R. et al. Stress, stress reduction and hypercholesterolemia in African Americans: a review. Ethn Dis, v. 9, p. 45।-62, 1999
5. CASIGLIA, E. et al. Arterial hypertension and mortality in the elderly. Amer J Hypertens, v. I I, p. 958-66, 2002

6. CRESPO, J.C. et al. The relationship of physical activity and body weight with all-cause mortality. Ann Epidemiol, v. 12 p. 543-52, 2002

7.DEMOSTHENES, B. Risk stratification of coronary heart disease in Greece: final results from the CARDIO 2000 Epidemiological Study. Prev Med, v. 35, n. 6, p. 548-56, 2002.

8. EAGLE, K.A.; HABER, E.; DESANCTIS, R.W. Cardiologia. 2.ed. Rio de Janeiro: Ed. Médica e Científica Ltda, 1993.

9. FORTI, A.C.; MARTINS, M.R.A. Diabetes mellitus em situações especiais. ILIB, v. 3, p. 21-7, 200। 
10. FORTI, N.; GIANNINI, S.D.; FUKUSHIMA, J. Coronary risk profile in Brazil. Atherosclerosis, v. I34, p. 156-7, 1997.

I I. FROVA, L. et al. Estimating population-based incidence and prevalence of major coronary events. J Clin Epidemiol, v. 5, p. 507-15, 1997.

12. GOYA, K. et al. Risk factors for asymptomatic atherosclerosis in Japanese type 2 diabetic patients without diabetic microvascular complications. Metabolism, v. 52, n. 10, p. 1302 6, 2003.

13. GUIMARÃES, A.C. Sobrepeso e obesidade: fatores de riso cardiovascular. Hipertensão, v. 4, p. 94-7, 2001.

14. HESLOP, P. et al. Change in job satisfaction and its association with self-reported stress, cardiovascular risk factors and mortality. Soc Sci Med, v. 54, p. 1589-99, 2002.

15. HESLOP, P. et al. Sleep duration and mortality: the effects of short or long sleep duration on cardiovascular and all-cause mortality in working men and women. Sleep Med, v. 3, p. 305-14, 2002.

16. KOHLMANN Jr., O. Tratamento anti-hipertensivo e proteção renal: importância do controle pressórico e do bloqueio dos sistemas renina-angiotensina. Hipertensão, v. 4, p. I01103, 2001.

17. KOON-SUNG et al. Review of the carotid and femoral intima-media thickness as an indicator of the presence of peripheral vascular disease and cardiovascular risk factors. Cardiovascular Rev, v. 54, n. 3, p. 528-38, 2002.

I 8. LAINSCAK, M.; KEBER I. Patient's view of heart failure: from the understanding to the quality of life. Europ J Cardiovascular Nursing, v. 2, n. 4, p. 275-8I, 2003.

19. LIMA, N.K. et al. Monitoração ambulatorial da pressão arterial (MAPA) no paciente idoso. Hipertensão, v. 4, p. 98-100, 2001.

20. LIPP, M.; ROCHA, J.C. Estresse, Hipertensão Arterial e Qualidade de Vida. São Paulo: Papirus Editora, 1996.

21. LIPP, M.N. et al. Como Enfrentar o Estresse. São Paulo: Ed. da Universidade de Campinas, 1986.

22. LIPPY, P. Aterosclerose: o novo ponto de vista. Sci Am, v. I, p. 54-63, 2002.

23. LOPES, I.I. et al. Apolipoproteína E, dislipidemia e aterosclerose. Dislipidemia Today, v. 2, p. 3-7, 2001.

24. LOURES-VALE,A.A.; MARTINEZ,T.M.R. Prevenção: importância e estratificação de risco. Atherosclerosis, v. 12, p. 7-12, 2001.

25. MacLEOD, J. et al. Are the effects of psychosocial exposures attributable to confounding. Evidence from a prospective observational study on psychological stress and mortality. Epidemiol Community Health, v. 55, p. 878-84, 2001.

26. MARTINEZ,T.Tratamento das dislipidemia em grupos especiais. ILIB, v. 3, p. I2-20, 200I.

27. McEVAN, S.R. et al. Heart and arterial disease risk factors measured in an office workforce: changes from 1992 to 1996. The Scottish Heart and Arterial Risk Prevention Group (SHARP). Scottish Med J, v. 43, p. 74-7, 1998.

28. MICHELON, E.; MORIGUCHI, E. Dislipidemias. Rev Bras Med, v. 56, p. I17-129, 1999.

29. MION Jr, D.; NOBRE, F. Risco Cardiovascular Global. São Paulo: Lemos Editorial, 2000.

30. MOTA, E.; MORIGUCHI, E. Dislipidemia em idosos: devemos tratar? Dislipidemia Today, v. I, p. 3-10, 200 I.
31. NEGRÃO, C.E. et al.Aspectos do treinamento físico na presença de pressão arterial. Hipertensão, v. 4, p. 84-7, 2001.

32. PALATINI, P.; JULIUS, S. The physiological determinants and risk correlations of elevated heart rate. Am J Hipertens, v. 12 , 3S-8S, 1999.

33. PORTO, C.C. Doenças do Coração: Prevenção e Tratamento. Rio de Janeiro: Ed. Guanabara Koogan, 1998.

34. RABELO, L.M. et al. Risk factors for atherosclerosis in students of a privative University in São Paulo, Brazil. Arq Bras Cardiol, v. 72, p. 569-80, 1999.

35. REBECCA, D.D. et al. Perceived stress following race-based discrimination at work is associated with hypertension in African Americans. The Metro Atlanta Heart Disease Study, 1999-200I. Soc Sci Med, v. 58, n. 3, p. 449-6I, 2004.

36. RECORD, N.B. et al. Mortality impact of an integrated community cardiovascular health program. Am J Prev Med, v. 19, p. 30-8, 2000.

37. ROGER, L.V. et al. Coronary disease surveillance in Olmsted County objectives and methodology.J Clin Epidemiol, v. 55, p. 593-601, 2002.

38. ROSEIN, G.E. et al. Influência do stress nos níveis sangüíneos de lipídeos, ácido ascórbico, zinco e outros parâmetros bioquímicos. Rev Anal Clin, v. 35, p. 19-25, 2003.

39. SAITO, I. et al. Trends in fatal coronary heart disease among people aged 25-74 years in Oita City, Japan, from 1987- 1998. J Clin Epidemiol, v. 55, p. 469-76, 2002.

40. SIRTORI, C.R.; GALLI, C. N-3 fatty acids and diabetes. Biomed Pharmacother, v. 56, p. 397-406, 2002.

4I. SOCIEDADE BRASILEIRA DE CARDIOLOGIA. Resumo das III Diretrizes Brasileiras sobre Dislipidemias e Diretriz de Prevenção da Aterosclerose do Departamento de Aterosclerose da Sociedade Brasileira de Cardiologia. Arq Bras Cardiol, v. 77, supl. III, p. I-4, 200 I.

42. SUGIYAMA, S. et al. Supersensitivity of atherosclerotic artery to constrictor effect of cigarette smoke extract. Cardiovascular Research, v. 38, p. 508-15, 1998.

43.TADDEI, C.F.G. et al. Estudo multicêntrico de idosos atendidos em ambulatórios de cardiologia e geriatria de Instituições brasileiras. Arq Bras Cardiol, v. 69, p. 327-33, 1997.

44.VÖGELE, C. Psychosomatic pathways to essential hypertension: the combined effect of anger and family history of cardiovascular disorders on cardiovascular reactivity. Int Cong Ser, v. I24I, p. 87-9, 2002.

45.WILHELMESEN, L. et al. Heart failure in the general population of men: morbidity, risk factors and prognosis. J Intern Med, v. 249, p. 253-61, 2001.

46. ZANCHETTI, A. The hipertensive patients with multiple risk factors. Is treatment really so difficult? Am J Hypertension, v. 10, 223S-229S, 1997.

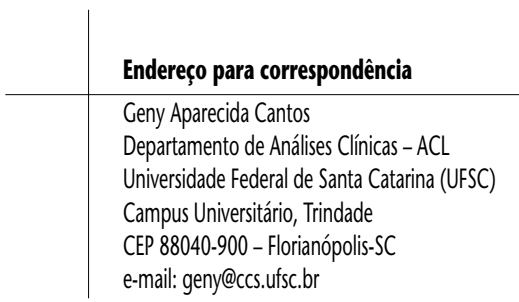

